\section{Aktive Erhaltungstherapie beim mCRC lohnt sich}

War die Behandlung eines metastasierten, kolorektalen Karzinoms (mCRC) mit Chemotherapie plus Bevacizumab erfolgreich, stellt sich die Frage nach der Erhaltungstherapie. In einer Studie der AIO wurden verschiedene Optionen verglichen.

\begin{abstract}
A $\mathrm{n}$ der Studie nahmen Patienten mit bisher nicht behandeltem mCRC und einem ECOG-Performancestatus von $0-2$ teil. Die über 24 Wochen geplante Induktionstherapie umfasste entweder Fluorouracil plus Folinsäure plus Oxaliplatin oder Capecitabin plus Oxaliplatin, jeweils ergänzt um Bevacizumab. Patienten ohne Progress wurden randomisiert zu einer Erhaltungstherapie mit einem Fluoropyrimidin plus Bevacizumab (Arm 1: $\mathrm{n}=158$ ), Bevacizumab alleine (Arm 2: $\mathrm{n}=156$ ) oder keiner Erhaltungstherapie (Arm 3: $\mathrm{n}=158$ ). Beim ersten Progress war eine Reinduktion mit der initialen Therapie geplant. Nach median 17 Monaten betrug die
\end{abstract}

Zeit bis zum Versagen der Therapiestrategie (zweiter Progress) 6,9 Monate (95\%-Konfidenzintervall [95\%-KI] 6,18,5) in Arm 1, 6,1 Monate (95\%-KI 5,3$7,4)$ in Arm 2 und 6,4 Monate (95\%-KI 4,8-7,6) in Arm 3. Bevacizumab alleine war damit der Kombination von Fluoropyrimidin/Bevacizumab als Erhaltungstherapie nicht unterlegen (Hazard Ratio [HR] 1,08; $95 \%$-KI 0,85-1,37), keine Erhaltungstherapie dagegen sehr wohl (HR 1,26; $95 \%$-KI 0,99-1,60).

Kritisch diskutieren die Forscher den gewählten Endpunkt „Versagen der vorgesehenen Therapiestrategie“, weil die im Protokoll vorgesehene Reinduktion beim ersten Progress relativ selten er- folgte (in Arm 1: 19\%, in Arm 2: $43 \%$ und in Arm 3: $46 \%$ ). Das lässt diese Strategie für den klinischen Alltag fraglich erscheinen.

Häufigste Grad-3-Nebenwirkung waren sensorische Neuropathien: darunter litten $13 \%$ der Patienten in Arm 1, 14\% in Arm 2 und $8 \%$ in Arm 3.

Fazit: Die Bevacizumab-Monotherapie zur Erhaltung war der Kombination Fluoropyrimidin/Bevacizumab nicht unterlegen. Dennoch empfehlen die Forscher eher den Einsatz der Kombination zur Erhaltung, um so im direkten Anschluss an die erfolgreiche Induktionstherapie und damit ohne Zeitverzug eine Erhaltungstherapie zur Krankheitskontrolle anzuschließen.

Friederike Klein

Hegewisch-Becker $\mathrm{S}$ et al. Maintenance strategies after first-line oxaliplatin plus fluoropyrimidine plus bevacizumab for patients with metastatic colorectal cancer (AIO 0207): a randomised, non-inferiority, open-label, phase 3 trial. Lancet Oncol. 2015;16(13):1355-69.

\section{Verstärkung der Chemotherapie verlängert Überleben beim mCRC}

Die Kombination zweier Zytostatika mit Bevacizumab ist ein häufig genutzter Standard beim metastasierten kolorektalen Karzinom (mCRC). Das Rückgrat der Chemotherapie weiter zu verstärken, kann sich lohnen.

$\mathrm{D}$ arauf weist eine aktuelle Analyse einer Phase-III-Studie hin, an der 508 Patienten mit nicht operablem mCRC (18-70 Jahre, ECOG-Performancestatus [ECOG-PS] $\leq 2$ oder $71-75$ Jahre, ECOG-PS 0) aus 34 italienischen Krebszentren teilnahmen. Sie erhielten Bevacizumab (5 mg/kg i.v.) plus randomisiert FOLFIRI $\left(180 \mathrm{mg} / \mathrm{m}^{2}\right.$ Irinotecan i. v. über $60 \mathrm{~min}$, gefolgt von $200 \mathrm{mg} / \mathrm{m}^{2}$ Folinsäure i.v. über $120 \mathrm{~min}, 400 \mathrm{mg} / \mathrm{m}^{2}$ Fluorouracil (5-FU) als Bolus i.v. sowie $2.400 \mathrm{mg} / \mathrm{m}^{2}$ 5-FU als 46 -h-Infusion) oder FOLFOXIRI $\left(165 \mathrm{mg} / \mathrm{m}^{2}\right.$ Irinotecan i.v. über $60 \mathrm{~min}$, gefolgt von $85 \mathrm{mg} /$ $\mathrm{m}^{2}$ Oxaliplatin i.v. und gleichzeitig 200 $\mathrm{mg} / \mathrm{m}^{2}$ Folinsäure über $120 \mathrm{~min}$, anschließend $3.200 \mathrm{mg} / \mathrm{m}^{2}$ 5-FU als 48 -hInfusion). Nach median 48,1 Monaten war das Gesamtüberleben (OS) im
FOLFOXIRI/Bevacizumab-Arm signifikant länger (median 29,8 vs. 25,8 Monate; Hazard Ratio [HR] 0,80, $95 \%$-Konfi-

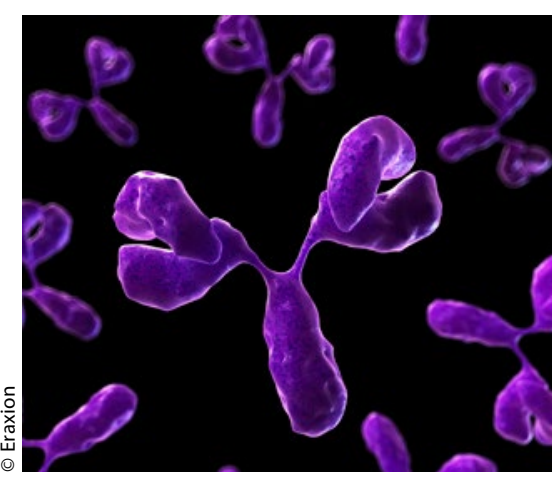

Auf die richtige Kombination von Antikörper- und Chemotherapieregime kommt es an. denzintervall $[95 \%-\mathrm{KI}] \quad 0,65-0,98$; $\mathrm{p}=0,03)$.

Es zeigte sich bei Patienten mit RASund BRAF-Wildtyp mit 37,1 Monaten ein längeres $O S$ als bei Patienten mit RAS-Mutation (25,6 Monate; HR 1,49, $95 \%$-KI 1,11-1,99) oder mit BRAF-Mutation (13,4 Monate; HR 2,79, $95 \%$-KI $1,75-4,46 ; \mathrm{p}<0,0001)$. Keine der molekularen Subgruppen profitierte aber von einer der beiden Therapien besonders $\left(p_{\text {interaction }}=0,52\right)$.

Fazit: Schon in der Primäranalyse war das progressionsfreie Überleben bei insgesamt beherrschbarer Toxizität nach FOLFOXIRI besser gewesen. Der jetzt belegte Gesamtüberlebensvorteil demonstriert deutlich, dass diese Therapie unabhängig vom RAS- oder BRAF-Mutationsstatus als Option für Patienten mit entsprechenden Einschlusskriterien zu berücksichtigen ist. Friederike Klein

Cremolini $C$ et al. FOLFOXIRI plus bevacizumab versus FOLFIRI plus bevacizumab as first-line treatment of patients with metastatic colorectal cancer: updated overall survival and molecular subgroup analyses of the open-label, phase 3 TRIBE study. Lancet Oncol. 2015;16(13):1306-15. 